\title{
Can Renal Parameters Predict the Mortality of Hospitalized COVID-19 Patients?
}

\author{
Eli Zolotov ${ }^{\mathrm{a}}$ Anat Sigal ${ }^{\mathrm{b}}$ Martin Havrda $^{\mathrm{a}}$ Karolína Jeřábkováa \\ Karolína Krátkáa, ${ }^{a}$ Nikola Uzlováa, b Ivan Rychlík ${ }^{a, b}$

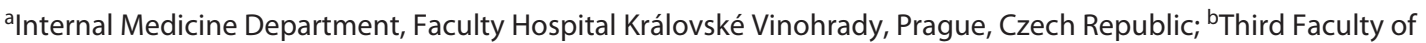 \\ Medicine, Charles University, Prague, Czech Republic
}

\section{Keywords}

Chronic kidney disease · Acute kidney injury · Coronavirus disease 2019 . Estimated glomerular filtration rate .

Prognosis

\begin{abstract}
Introduction: Our study aimed to analyze whether renal parameters can predict mortality from COVID-19 disease in hospitalized patients. Methods: This retrospective cohort includes all adult patients with confirmed COVID-19 disease who were consecutively admitted to the tertiary hospital during the 4-month period (September 1 to December 31, 2020). We analyzed their basic laboratory values, urinalysis, comorbidities, length of hospitalization, and survival. The RIFLE and KDIGO criteria were used for AKI and CKD grading, respectively. To display renal function evolution and the severity of renal damage, we subdivided patients further into 6 groups as follows: group 1 (normal renal function), group 2 (CKD grades $2+3 a)$, group 3 (AKI-DROP defined as whose $\mathrm{s}-\mathrm{Cr}$ level dropped by $>33.3 \%$ during the hospitalization), group 4 (CKD 3b), group 5 (CKD $4+5)$, and group 6 (AKI-RISE defined as whose $\mathrm{s}-\mathrm{Cr}$ level was elevated by $\geq 50 \%$ within 7 days or by $\geq 26.5 \mu \mathrm{mol} / \mathrm{L}$ within $48 \mathrm{~h}$ during hospitalization).
\end{abstract}

Then, we used eGFR on admission independently of renal damage to check whether it can predict mortality. Only 4 groups were used: group I - normal renal function (eGFR > $1.5 \mathrm{~mL} / \mathrm{s}$ ), group II - mild renal involvement (eGFR 0.75-1.5), group III - moderate (eGFR 0.5-0.75), and group IV - severe (GFR <0.5). Results: A total of 680 patients were included in our cohort; among them, 244 patients displayed normal renal function, 207 patients fulfilled AKI, and 229 patients suffered from CKD. In total, a significantly higher mortality rate was found in the AKI and the CKD groups versus normal renal function $-37.2 \%$ and $32.3 \%$ versus $9.4 \%$, respectively $(p<$ 0.001 ). In addition, the groups 1-6 divided by severity of renal damage reported mortality of $9.4 \%, 21.2 \%, 24.1 \%, 48.7 \%$, $62.8 \%$, and $55.1 \%$, respectively $(p<0.001)$. The mean hospitalization duration of alive patients with normal renal findings was 9.5 days, while it was 12.1 days in patients with any renal damage $(p<0.001)$. When all patients were compared according to eGFR on admission, the mortality was as follows: group I (normal) $9.8 \%$, group II (mild) $22.1 \%$, group III (moderate) $40.9 \%$, and group IV (severe) $50.5 \%$, respectively $(p<0.001)$. It was a significantly better mortality predictor than CRP on admission (AUC 0.7053 vs. 0.6053). Conclu-

Eli Zolotov and Anat Sigal contributed equally.
Karger@karger.com www.karger.com/kbr

Karger $\stackrel{\text { ' }}{5}$

GOPEN ACCESS
(C) 2022 The Author(s)

Published by S. Karger AG, Basel

This is an Open Access article licensed under the Creative Commons Attribution-NonCommercial-4.0 International License (CC BY-NC) (http://www.karger.com/Services/OpenAccessLicense), applicable to the online version of the article only. Usage and distribution for commercial purposes requires written permission.
Correspondence to:

Ivan Rychlík, ivan.rychlik@fnkv.cz 
sions: Mortality in patients with abnormal renal function was 3 times higher compared to patients with normal renal function. Also, patients with renal damage had a worse and longer hospitalization course. Lastly, eGFR on admission, independently of renal damage type, was an excellent tool for predicting mortality. Further, the change in $\mathrm{s}-\mathrm{Cr}$ levels during hospitalization reflected the mortality prognosis.

(c) 2022 The Author(s).

Published by S. Karger AG, Basel

\section{Introduction}

In December 2019, a cluster of pneumonia cases in Wuhan, China, led to the discovery of severe acute respiratory syndrome coronavirus 2 (SARS-CoV-2) [1]. After SARS and the Middle East respiratory syndrome, it is the third coronavirus-caused outbreak in the 21 st century. From Wuhan, the disease spread quickly to other parts of China and other countries. The WHO announced coronavirus disease 2019 (COVID-19) a global pandemic, on March 11, 2020. As of January 2022, more than 290 million people were infected and more than 5 million died worldwide [2].

According to large-scale epidemiological evidence, the mortality risk of SARS-CoV-2 infection is considerably higher for older and comorbid chronic disorders such as coronary disease, bronchial asthma, chronic respiratory diseases, arterial hypertension, and cancer. In addition, a history of kidney dysfunctions (chronic) or new mild to severe dysfunctions (acute) was discovered to significantly increase the mortality in COVID-19 patients independent of other factors $[3,4]$.

One striking feature of this disease is its variability in clinical outcome, ranging from inapparent infection to hospitalization for those individuals with sufficient disease burden and comorbid conditions, even with a high risk of mortality. Different factors predictive of COVID-19 clinical course and outcomes were identified. Still, currently it is accepted that findings associated with increased disease severity and/or mortality include age $>55$ years, multiple pre-existing comorbidities, hypoxia, specific computed tomography findings indicative of extensive lung involvement, diverse laboratory test abnormalities, and biomarkers of end-organ dysfunction [5].

However, a simple and easy-to-use model predicting mortality and based on data easily available to clinicians in admission to the hospital is lacking, although it would help direct limited medical and personnel resources toward those patients at greater risk of dying
[6]. Thus, our goal was to check whether renal predictors of mortality are useful in order to be able to evaluate correctly the prognosis of the patient warranting hospitalization.

\section{Methods}

\section{Participants and Documentations}

This retrospective cohort study reviewed all patients admitted to the tertiary university hospital (University Hospital Královské Vinohrady, FNKV) with a catchment area of about 350 thousand inhabitants of the city of Prague due to suspected or confirmed COVID-19 disease. All such consecutive patients who required hospitalization between September 1, 2020, and December 31, 2020 , were included. This period represented the second "wave" of COVID-19 disease in the Czech Republic.

Using the patients' electronic database of the FNKV, we included all patients who displayed SARS-CoV-2 reverse transcriptase polymerase chain reaction (PCR) test positivity on admission taken by standard throat swab and who were clinically suspected or symptomatic due to the COVID-19 disease what actually was the reason for hospitalization. Further, patients admitted to the hospital for other reasons than COVID-19 disease and who became PCR positive during the hospitalization were included in the cohort as well. All the patients were adults (more than 18 years old). Patients who had a negative PCR test for COVID-19, all asymptomatic patients (admitted for any reason other than COVID-19), and 3 patients who had no data at all (no history and no present lab tests) were excluded.

Further, we rechecked all our data using the National Information System of Infectious Diseases (ISIN) system. This is a countrywide centralized system managed by the Ministry of Health. All physicians in the Czech Republic are obliged to document the COVID-19 disease for all patients treated in their units or departments. The ISIN system gets updated each day. Thus, all hospitalized patients with proven PCR tests are reported, including their oxygen treatment, hospitalization length, and their outcome (alive/death). These national data helped us confirm our data regarding the number of COVID-19 symptomatic patients, their therapy, and their length of hospitalization.

Using the electronic medical record (EMR), the following data were extracted:

1. The patients' comorbidities: obesity (defined by BMI >30), arterial hypertension (patients who underwent antihypertensive treatment), coronary artery diseases (patients who underwent any procedure associated with their coronary arteries), diabetes mellitus (DM) (patients who were undergoing any kind of medical treatment for DM), dyslipidemia (documented in their charts), bronchial asthma (recorded in their charts), and chronic obstructive pulmonary disease (recorded in their charts);

2. The basic lab values and the urinalysis: i.e., serum creatinine (s-Cr) levels and CRP on admission, estimated glomerular filtration rate (eGFR) on admission using the CKD-EPI equation (Chronic Kidney Disease Epidemiology Collaboration) [7], and maximal levels of strip proteinuria and strip hematuria during hospitalization. The proteinuria and hematuria groups were divided into four subgroups according to the amount of protein and red blood cells in the urine. In each subgroup, the 


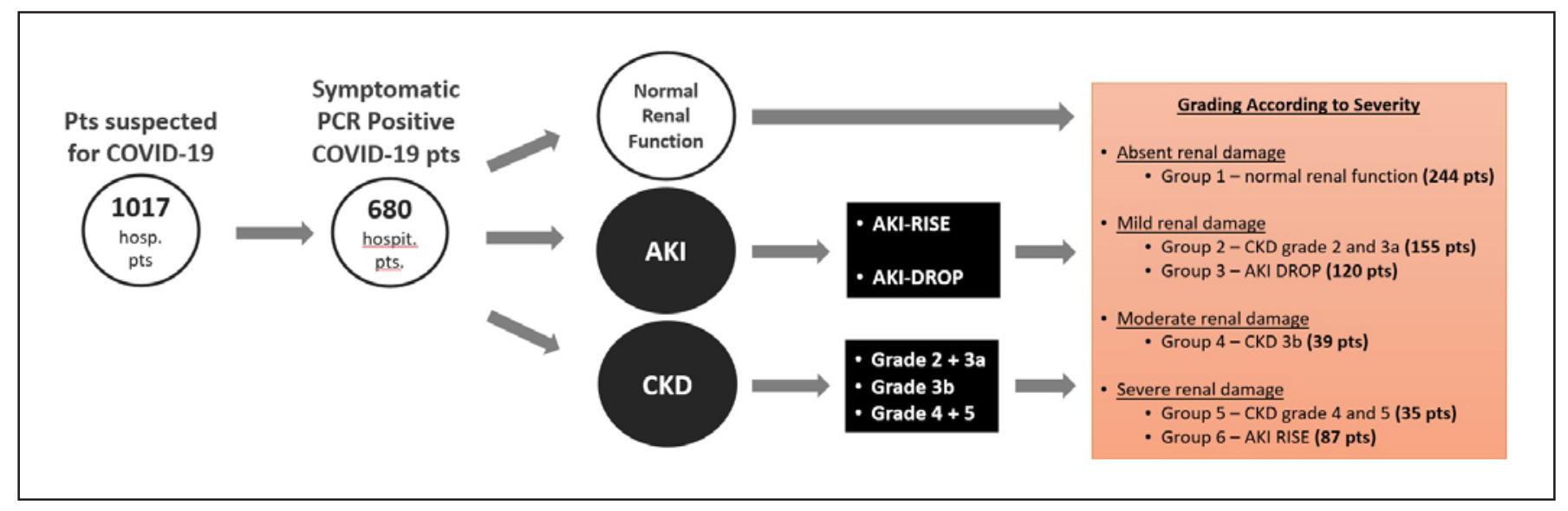

Fig. 1. Patients' flowchart. A total of 1,017 suspected patients for COVID-19 were analyzed, and 680 symptomatic confirmed PCR-positive COVID-19 patients were included in our cohort. This group was further divided into $\mathrm{AKI}, \mathrm{CKD}$, and normal renal function groups. The $\mathrm{AKI}$ and the CKD groups were subdivided further into "AKI-RISE," "AKI-DROP," and into CDK grades 2, 3a, 3b, 4, and 5. These groups were done in order to form the final useful classification. A total of 6 groups were formed according to the severity of renal damage.

mortality was documented in the patients with normal renal function, AKI, or CKD;

3. Further data concerning the course of hospitalization: i.e., the length of the hospitalization (in days), the survival (dead or alive), and the type of oxygen therapy (no therapy needed, nasal oxygen, high-flow nasal oxygen/HFNO/mechanical ventilation);

4. Patients with AKI or CKD (see definition below).

\section{Definitions and Grouping}

Patients were divided into three groups: normal renal findings, AKI (acute kidney injury), and CKD (chronic kidney disease). However, to display better the renal function evolution and the severity of renal damage, we subdivided patients further according to the following criteria:

1. The order for AKI definition based on standard RIFLE criteria [8] was used: first, if the s-Cr level rose by $\geq 50 \%$ within 7 days during hospitalization, they were classified as "AKI-RISE." Then, a drop of s-Cr level during hospitalization by $>33.3 \%$ (as a reflection of undetected previous rise by $>50 \%$ ) was classified as “AKI-DROP." Lastly, a raise of s-Cr level during hospitalization by $\geq 26.5(\mu \mathrm{mol} / \mathrm{L})$ within $48 \mathrm{~h}$ was added to the " $A K I$ RISE" group.

We divided the AKI groups into "AKI-RISE” and "AKI-DROP” because we hypothesized that the mortality might differ between these groups. Moreover, using the AKI RIFLE definition solely may lead to miss patients with AKI. Patients admitted with already s-Cr level at its highest peak may be missed since their s-Cr level rise will not be documented in the EMR (since it occurred outside the hospital).

2. Then, we checked patients who were not part of the AKI group. We measured their eGFR, and those who had abnormal eGFR of $<1.5\left(\mathrm{~mL} / \mathrm{s} / 1.73 \mathrm{~m}^{2}\right)$ during their whole period of hospitalization were classified as part of the CKD group. Patients who had at least one documented normal eGFR $\geq 1.5\left(\mathrm{~mL} / \mathrm{s} / 1.73 \mathrm{~m}^{2}\right)$ were re- moved from this group. Furthermore, this group was divided into CKD grades using standard grading according to the KDIGO eGFR definition [9] (groups CKDG1-CKDG5). For the CKD grouping, we preferred to use the eGFR solely and not the charts to avoid missing patients who do not have any documentation about their kidney status. In addition, some patients are not part of the CKD group by the medical charts, even though they have a low eGFR due to their age and gender. Therefore, our definition helped us to include all the patients with abnormal renal function without missing any single patient.

3. Patients who did not meet any of the criteria above (not AKI or CKD) were classified as those with normal renal function.

Thus, finally, to investigate the patient's mortality, according to the severity and type of renal damage, we formed 6 groups: group 1 (normal renal function), group 2 (CKD 2 and CKD 3a), group 3 (AKI-DROP), group 4 (CKD 3b), group 5 (CKD 4 and CKD 5), and group 6 (AKI-RISE), i.e., the groups $2-3$ represented mild renal damage, while the groups 4-6 represented severe renal impairment (shown in Fig. 1).

Moreover, independently of the type of renal damage, we tested if only eGFR value (CKD-EPI formula) on admission can predict mortality. For this reason, only four groups were formed: group $I$ - normal renal function (eGFR > $1.5\left[\mathrm{~mL} / \mathrm{s} / 1.73 \mathrm{~m}^{2}\right]$ ), group II - mild renal involvement (eGFR 0.75-1.5 [mL/s/1.73 $\left.\left.\mathrm{m}^{2}\right]\right)$, group III - moderate (eGFR $0.5-0.75\left[\mathrm{~mL} / \mathrm{s} / 1.73 \mathrm{~m}^{2}\right]$ ), and group $I V$ - severe $\left(\mathrm{eGFR}<0.5\left[\mathrm{~mL} / \mathrm{s} / 1.73 \mathrm{~m}^{2}\right]\right)$. To better understand the importance of eGFR as a predictor of mortality, we compared our results to the correlation of the CRP (on admission as well) to mortality.

\section{COVID-19 Therapy - General Rules}

During the entire period of this study, we used the WHO guidelines for the therapy of COVID-19 disease in our hospital [10]. All patients with the indication of any oxygen supplementation (= patients suffering from hypoxia) received corticosteroids. It was shown 
Table 1. Demographics and comorbidities

\begin{tabular}{|c|c|c|c|c|}
\hline & Total & $\begin{array}{l}\text { Normal } \\
\text { renal function }\end{array}$ & $\begin{array}{l}\text { Acute } \\
\text { kidney injury }\end{array}$ & $\begin{array}{l}\text { Chronic } \\
\text { kidney disease }\end{array}$ \\
\hline \multicolumn{5}{|c|}{ Demographics and profile } \\
\hline Patients, $n$ & 680 & 244 & 207 & 229 \\
\hline Mean age, years & 72.5 & 67.4 & 76.4 & 79.7 \\
\hline Male sex, $n(\%)$ & $363(53.3)$ & $135(55.3)$ & $113(54.6)$ & $115(50.2)$ \\
\hline Female sex, $n(\%)$ & $317(46.7)$ & $109(44.7)$ & $94(45.4)$ & $114(49.8)$ \\
\hline \multicolumn{5}{|l|}{ Comorbidities, $n(\%)$} \\
\hline Diabetes mellitus & $215(31.6)$ & $46(18.8)$ & $86(41.5)$ & $83(36.2)$ \\
\hline Obesity & $82(12.6)$ & $26(10.6)$ & $36(17.3)$ & $20(8.7)$ \\
\hline $\begin{array}{l}\text { Coronary artery } \\
\text { disease }\end{array}$ & $134(19.7)$ & $21(8.6)$ & $49(23.6)$ & $64(27.9)$ \\
\hline Hypertension & $454(66.7)$ & $121(49.5)$ & $150(72.4)$ & $183(79.9)$ \\
\hline Dyslipidemias & 217 (31.9) & $65(26.6)$ & $68(32.8)$ & $84(36.6)$ \\
\hline COPD & $55(8)$ & $15(6.1)$ & $18(8.7)$ & $22(9.6)$ \\
\hline Asthma & $54(7.9)$ & $29(11.8)$ & $14(6.7)$ & $11(4.8)$ \\
\hline
\end{tabular}

COPD, chronic obstructive pulmonary disease.

to decrease mortality [11]. The other common part of therapy was the use of antiviral therapy represented by remdesivir for patients with COVID-pneumonia requiring oxygen therapy but not on mechanical ventilation. Some but few patients got COVID-19 convalescent plasma during their first $72 \mathrm{~h}$ of hospitalization. Concerning $\mathrm{O}_{2}$ supportive therapy, simple nasal $\mathrm{O}_{2}$ and HFNO therapy were available for all patients within their needs. However, concerning mechanical ventilation, the limitations of therapy (using Ethics Committee-approved rules) were applied. The other medications and drugs (i.e., diuretics and antibiotics) were used according to usual therapeutical standards in our hospital.

\section{Statistics}

The statistical analysis was done using the software STATISTICA 12. Continuous variables were reported as means with standard deviation, and the 2 sample $t$ test was used to compare groups. Categorical variables were reported as proportions, and Pearson's $\chi^{2}$ test was used to compare groups. Multivariate logistic regression was used to determine an adjusted influence of variables on outcome. The results of multivariate analyses were expressed as odds ratios with 95\% confidence interval, the area under curve (AUC), and $p$ value. All tests were performed at the $5 \%$ level of significance.

\section{Results}

\section{Patients' Characteristics and Grouping}

We analyzed altogether 1,017 patients who were suspected for or positive to COVID-19. After excluding patients using the criteria mentioned earlier, we ended up with 680 symptomatic confirmed PCR-positive COVID-19 patients included in our cohort.

The AKI group contained 207 patients, the CKD group contained 229 patients, and 244 patients had a normal renal function. The "AKI-RISE" group included 87 pa-
Table 2. Mortality by the severity of the renal damage $(p<0.001)$

\begin{tabular}{lll}
\hline & Total & Mortality, \% \\
\hline $\begin{array}{l}\text { Absent renal damage } \\
\quad \text { Group 1 - normal renal function }\end{array}$ & 244 & 9.4 \\
$\begin{array}{l}\text { Mild renal damage } \\
\quad \text { Group 2 - CKD grades 2 and 3a }\end{array}$ & 155 & 21.2 \\
$\quad$ Group 3 - AKI-DROP & 120 & 24.1 \\
$\begin{array}{l}\text { Moderate renal damage } \\
\quad \text { Group 4 - CKD 3b }\end{array}$ & 39 & 48.7 \\
$\begin{array}{l}\text { Severe renal damage } \\
\quad \text { Group 5 - CKD grades 4 and 5 } \\
\text { Group 6 - AKI-RISE }\end{array}$ & 35 & 62.8 \\
& 87 & 55.1 \\
\hline
\end{tabular}

tients, and the "AKI-DROP" group contained 120 patients. The composition of the CKD group sorted by eGFR was as follows: CKD-G2 (grade 2) contained 105 patients, G3a contained 50 patients, G3b contained 39 patients, G4 contained 25 patients, and G5 contained 10 patients (shown in Fig. 1).

The median age of all the patients was 75 years, while in the normal renal function group, AKI group, and the CKD groups, the median age was $64.5,77.3$, and 81.1 years, respectively. $53.3 \%$ of all the patients were males, while $55.3 \%, 54.5 \%$, and $50.2 \%$ were males in the normal renal function, AKI, and CKD group, respectively. The patients' comorbidities were distributed in the following manner: $66.7 \%$ had hypertension, $31.9 \%$ had dyslipidemia, $31.6 \%$ had DM, $19.7 \%$ had coronary artery diseases, $12.0 \%$ had obesity, $8.1 \%$ had chronic obstructive pulmonary disease, and 7.9\% had bronchial asthma. All patients' 
Table 3. Multivariable analysis

\begin{tabular}{lllc}
\hline & OR & $95 \%$ Cl & $p$ value \\
\hline Age, years & 1.05 & $1.03-1.08$ & $<\mathbf{0 . 0 0 1}$ \\
Gender (male) & 1.62 & $1.07-2.43$ & $\mathbf{0 . 0 2}$ \\
Renal groups & & & \\
$\quad$ Group 1 - normal renal function & Ref & Ref & Ref \\
Group 2 - CKD grades 2 and 3a & 1.26 & $0.66-2.40$ & 0.46 \\
Group 3 - AKI-DROP & 1.61 & $0.83-3.12$ & 0.15 \\
Group 4 - CKD 3b & 3.68 & $1.57-8.62$ & $\mathbf{0 . 0 0 2}$ \\
Group 5 - CKD grades 4 and 5 & 7.64 & $3.20-18.20$ & $<\mathbf{0 . 0 0 1}$ \\
Group 6 - AKI-RISE & 6.29 & $3.28-12.05$ & $<\mathbf{0 . 0 0 1}$ \\
Comorbidities & & & \\
$\quad$ Coronary artery disease & 0.90 & $0.56-1.45$ & 0.63 \\
$\quad$ Diabetes mellitus & 1.18 & $0.77-1.80$ & 0.43 \\
$\quad$ Obesity & 1.40 & $0.75-2.63$ & 0.28 \\
$\quad$ Hypertension & 0.94 & $0.59-1.50$ & 0.80 \\
$\quad$ Dyslipidemia & 0.84 & $0.55-1.30$ & 0.44 \\
COPD & 1.83 & $0.95-3.53$ & 0.06 \\
$\quad$ Asthma & 0.78 & $0.32-1.91$ & 0.59 \\
\hline
\end{tabular}

$\mathrm{OR}$, odds ratio; $\mathrm{Cl}$, confidential interval, Ref, reference values; CKD, chronic kidney disease; $\mathrm{AKI}$, acute kidney injury; COPD, chronic obstructive pulmonary disease. Significant $p$ values $(<0.05)$ are given in bold.

characteristics and comorbidities are given in detail in Table 1.

In the $\mathrm{AKI}$ and the $\mathrm{CKD}$ groups, respectively, a higher percentile of DM (41.5\% and $36.2 \%)$, obesity (17.3\% and $8.7 \%)$, coronary artery diseases (23.6\% and $27.9 \%)$, dyslipidemia (32.8\% and 36.6\%), and hypertension $(72.4 \%$ and $79.9 \%)$ was observed. Nevertheless, bronchial asthma was more prominent in the normal renal function group (11.9\%).

\section{Hospitalization Course and Mortality by Severity of the Renal Disease}

After analyzing all collected data, we found that the mortality was higher in the AKI and in the CKD groups than those with normal kidney function $-37.2 \%$ and $32.3 \%$ versus $9.4 \%$, respectively ( $p<0.001$ ). When evaluating the mortality using the 6 group distribution which indicates on the severity of the renal damage, we found an increase in mortality in groups with more severe renal damage-groups 4, 5, and 6 (see Table 2). Mortality risk was in concordance with the severity of kidney damage given in groups $1-6(p<0.001)$.

In the multivariable analysis, we evaluated the same groups as well as the age, sex, and any comorbidity (see Table 3). The worse prognosis was significantly connect-

Mortality of Hospitalized COVID-19

Patients and Renal Parameters
Table 4. Hematuria and proteinuria

\begin{tabular}{|c|c|c|}
\hline & $N$ & Mortality, \% \\
\hline \multicolumn{3}{|c|}{ Proteinuria } \\
\hline \multicolumn{3}{|c|}{ Normal renal function } \\
\hline+0 & 41 & 4.8 \\
\hline+1 & 91 & 9.8 \\
\hline+2 & 23 & 8.7 \\
\hline$\geq+3$ & 3 & 33.3 \\
\hline \multicolumn{3}{|l|}{ AKI } \\
\hline+0 & 11 & 45.4 \\
\hline+1 & 72 & 22.2 \\
\hline+2 & 48 & 41.6 \\
\hline$\geq+3$ & 11 & 45.4 \\
\hline \multicolumn{3}{|l|}{ CKD } \\
\hline+0 & 14 & 14.2 \\
\hline+1 & 99 & 29.2 \\
\hline+2 & 38 & 42.1 \\
\hline$\geq+3$ & 8 & 37.5 \\
\hline \multicolumn{3}{|l|}{ Total } \\
\hline+0 & 66 & 13.64 \\
\hline+1 & 262 & 20.6 \\
\hline+2 & 109 & 34.8 \\
\hline$\geq+3$ & 22 & 40.9 \\
\hline \multicolumn{3}{|c|}{ Hematuria } \\
\hline \multicolumn{3}{|c|}{ Normal renal function } \\
\hline+0 & 109 & 9.1 \\
\hline+1 & 10 & 0 \\
\hline+2 & 14 & 7.1 \\
\hline$\geq+3$ & 25 & 12 \\
\hline \multicolumn{3}{|l|}{ AKI } \\
\hline+0 & 43 & 25.5 \\
\hline+1 & 15 & 40 \\
\hline+2 & 28 & 28.5 \\
\hline$\geq+3$ & 56 & 37.5 \\
\hline \multicolumn{3}{|l|}{$\mathrm{CKD}^{-}$} \\
\hline+0 & 64 & 18.7 \\
\hline+1 & 23 & 39.1 \\
\hline+2 & 33 & 39.3 \\
\hline$\geq+3$ & 39 & 41 \\
\hline \multicolumn{3}{|l|}{ Total } \\
\hline+0 & 216 & 15.2 \\
\hline+1 & 48 & 31.2 \\
\hline+2 & 75 & 29.3 \\
\hline$\geq+3$ & 120 & 33.3 \\
\hline
\end{tabular}

ed to age, male gender, and severe renal damage ( $\mathrm{CKD} \geq$ G3b, AKI-RISE). However, we did not find a significant connection with any of the listed comorbidities.

To check the disease course, we also examined the mean hospitalization duration in alive patients who had a normal renal function and in alive patients who have any kind of kidney damage (independent of AKI or CKD status). We found that the hospitalization duration was 9.5 days versus 12.1 days, respectively $(p<0.001)$.

Kidney Blood Press Res 2022;47:309-319 


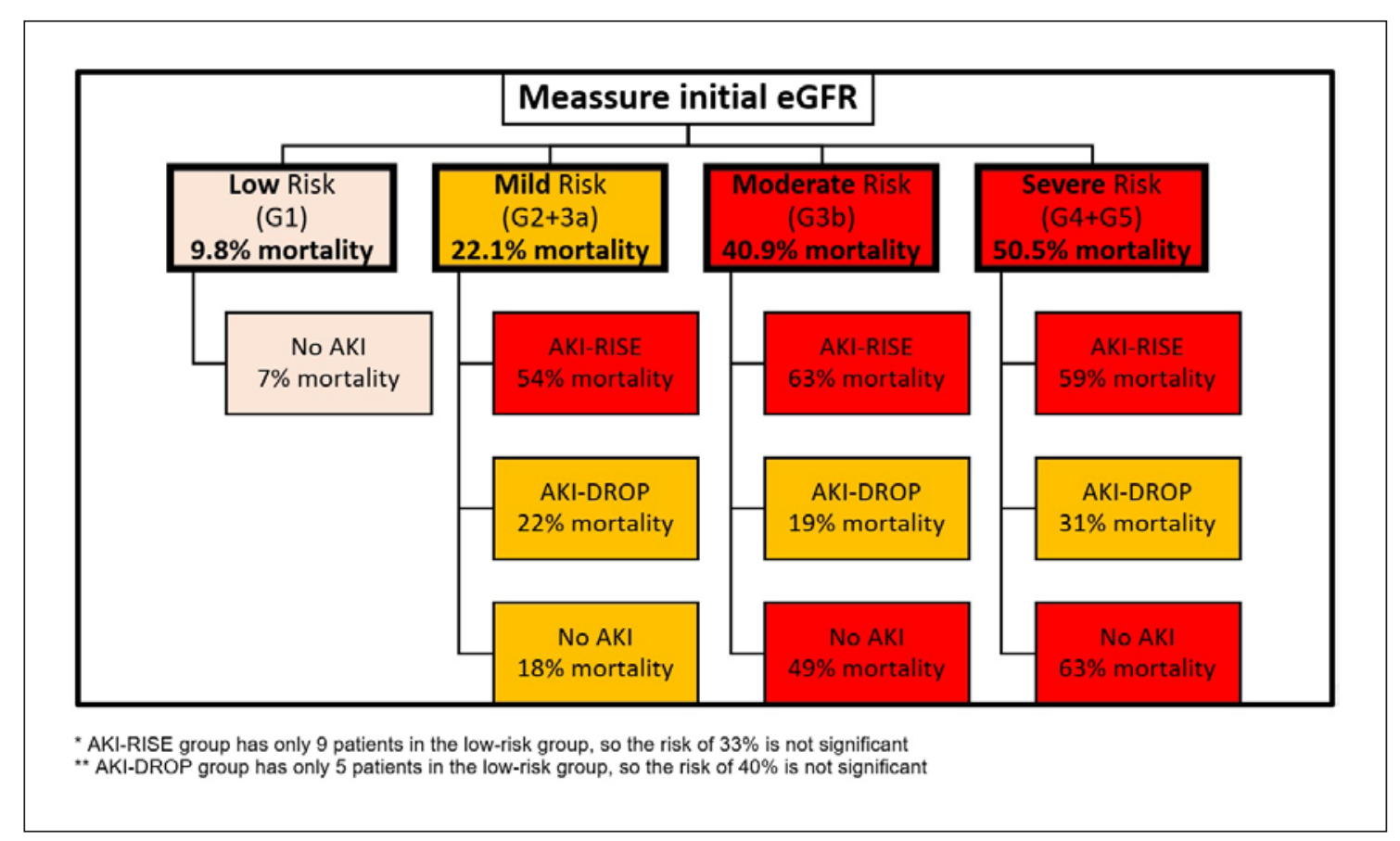

Fig. 2. Mortality prediction diagram. After using the eGFR calculator for assessing the mortality risks, we found that the prognosis may change depending on 3 groups: "AKI-RISE" (s-Cr rise), "AKI-DROP” (s-Cr drop), and patients without $A K I$ (no change in s-Cr - normal renal function and CKD). The figure shows how the initial risk of mortality changes depending on these groups according to the change in s-Cr level (rise/drop/no change). The AKI-RISE group has only 9 patients in the low-risk group, so the risk of $33 \%$ is not significant. The AKI-DROP group has only 5 patients in the low-risk group, so the risk of $40 \%$ is not significant.

Additionally, using a strip test, we recorded the levels of proteinuria and hematuria, in 459 patients during hospitalization. The detailed mortality rate distribution in each group is shown in Table 4. A correlation was found between the severity of proteinuria and mortality. It is of interest that this was truly independent of renal function. Nevertheless, for hematuria, only the CKD group displayed the same.

\section{Renal Parameters as Predictors of Mortality}

We measured the eGFR on admission for all patients in the cohort. In the logistic regression model, eGFR on admission was a significant predictor of mortality (AUC $0.7053, p<0.01$ ). Then, as explained above, we divided patients into 4 groups: group I (grade 1), group II (grade $2+3 a$ ), group III (grade $3 b$ ), and group IV (grade $4+5$ ). We found that independent of their renal status, the mortality was $9.8 \%, 22.1 \%, 40.9 \%$, and $50.5 \%$, respectively $(p<0.001)$. In other words, the eGFR calculator can work as a great predictor of mortality.
After using eGFR and finding the initial mortality risk of each group, we found that the prognosis may be changed according to the fluctuation of s-Cr level during the hospitalization. Therefore, we formed 3 groups: "AKIRISE," "AKI-DROP," and "NO AKI" (normal renal function and CKD) (shown in Fig. 2).

We can see that patients who had an initial severe risk (group IV, i.e., grade 4 and grade 5) with a mortality rate of $50.5 \%$, whose s-Cr level dropped during hospitalization, had a new prognosis of $31 \%$ mortality. However, on the contrary, if their s-Cr level was stable ("NO AKI" group) or it went up (“AKI-RISE" group), the mortality did not change significantly $-63 \%$ and $59 \%$, respectively.

Patients with moderate risk (group III, i.e., grade $3 \mathrm{~b}$ ) had a similar trend. In the "AKI-DROP" group, the mortality dropped to $19 \%$, while in the "NO $A K I$ " group and the "AKI-RISE" group, the mortality reached $49 \%$ and $63 \%$, respectively.

On the contrary, if the initial risk was mild, an increase in s-Cr ("AKI-RISE") level increases the mortality from $22.1 \%$ to $54 \%$. Furthermore, if the s-Cr level stays stable 
Fig. 3. The correlation between eGFR and CRP values on admission.

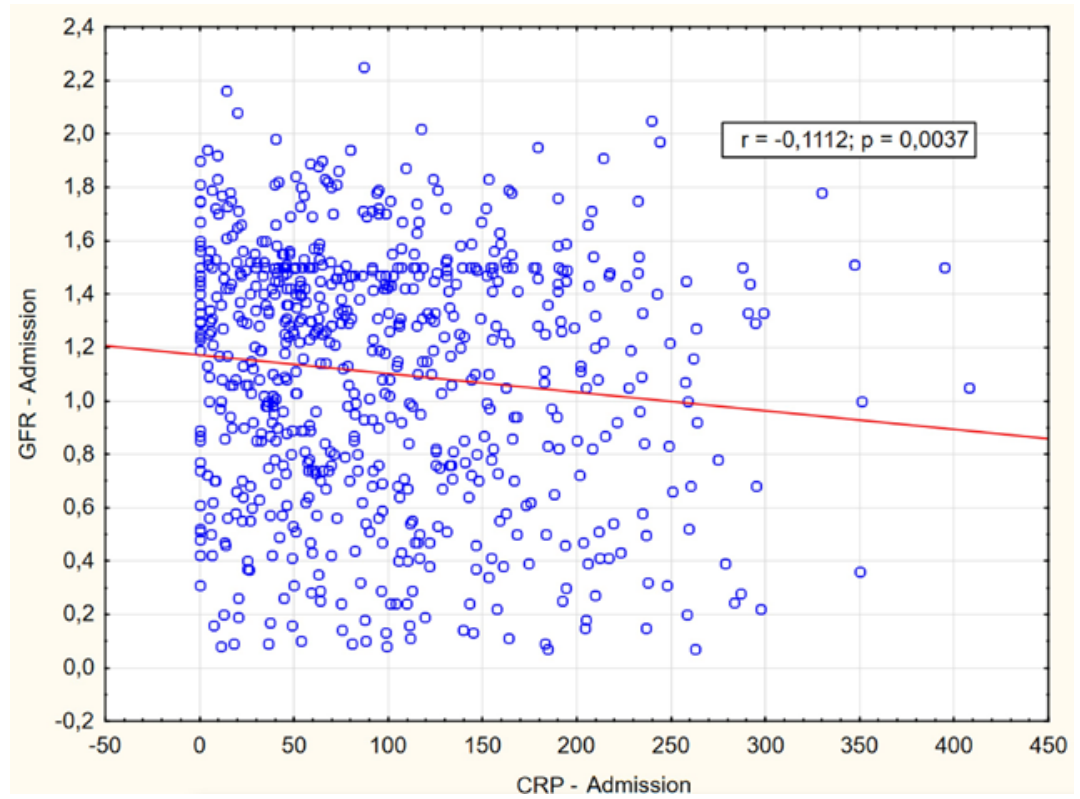

Fig. 4. Prediction of patient's death risk during hospitalization, which is assessed by the eGFR value on admission.

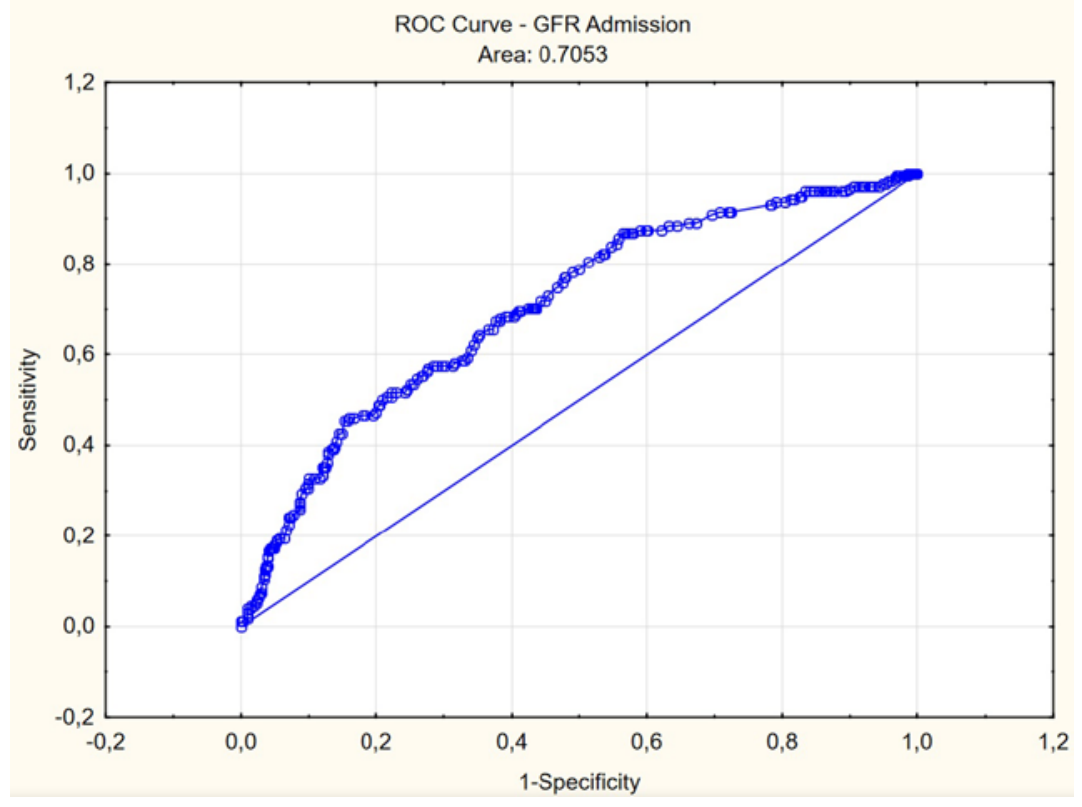

(“NO AKI") or even it dropped down (“AKI-DROP”), the mortality does not significantly change $-18 \%$ and $22 \%$, respectively.

Multiple studies have already shown that s-CRP may be a good predictor of mortality from COVID-19 [12]. Therefore, we decided to collect the CRP level on admission (it was available in all patients in the interval from
-1 to +3 days from admission) and evaluate its possible correlation with eGFR and, further, its ability to predict mortality. The correlation coefficient between eGFR and CRP reaches the value $r=-0.1112(p=0.0037)$, i.e., this is a very weak indirect correlation between these two parameters (shown in Fig. 3). Furthermore, we evaluated the ability of CRP to predict mortality versus eGFR by 
Fig. 5. Prediction of patient's death risk during hospitalization, which is assessed by the CRP value on admission.

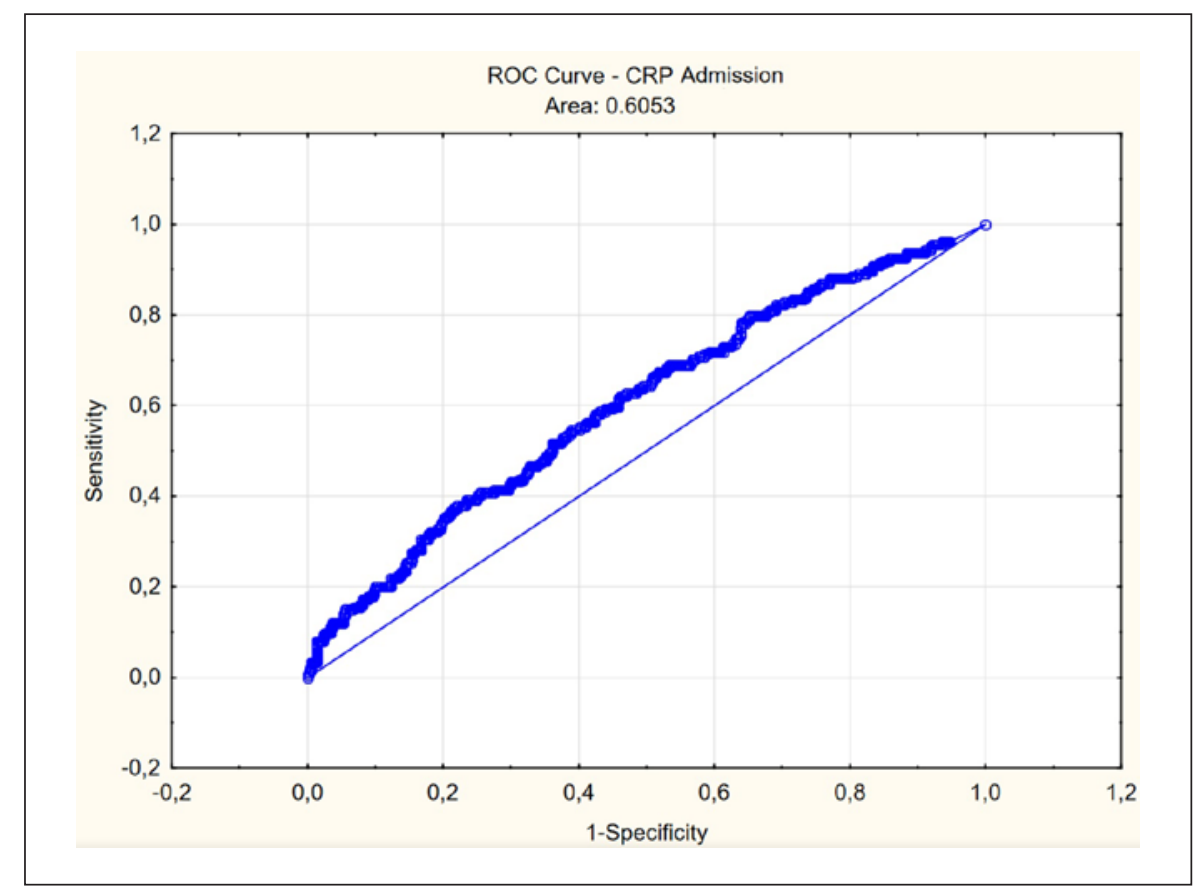

using the AUC method in the logistic regression model. We confirmed that the predictive power of the eGFR parameter is significantly higher (AUC $=0.7053$ ) than the CRP parameter (AUC $=0.6053$ ) (shown in Fig. 4 and Fig. 5).

\section{Discussion}

Our study is the first retrospective cohort study conducted in the Czech Republic that evaluates the renal damage's impact on mortality from SARS-CoV-2 infection. We proved that if the disease conditions require hospitalization, patients suffering from any kind of renal damage (AKI or CKD) bear a more than three times higher risk for mortality than those with normal renal function. Even though some studies show that mortality and worse COVID-19 course depends on the renal damage [13], on CKD stages [4], and on lab values, we believe that we bring some new knowledge in this matter, particularly from the "real-life" point of view.

One important feature of our study was that all consecutive patients requiring hospitalization were enrolled in the study. The ISIN system's use made the collected information much more accurate than solely relying on the EMR. No patients were missed, and even more, all the oxygen therapies could be evaluated, too. Besides, being a large tertiary hospital allowed us to handle a bigger sample size and include a larger scale of various patients' comorbidities. This fact allowed us to evaluate the complexity of the comorbidities and their influence on the COVID-19 prognosis in greater detail than other studies [3].

However, since we aimed to analyze the situation in "real-life" conditions in the Emergency department, we had to quantify kidney damage when limited patient's history and lab data were available. While most other studies [14] used two groups - AKI or CKD, and they referred them as two distinct entities, we decided to formulate a slightly different grouping system. It helped us reflect on the current severity of renal damage rather than its acute/chronic origin. Furthermore, we tried to use a simple classification, which would be more beneficial for the physician to predict the life prognosis of the patients at the time of hospital admission.

First, however, we had to formulate a relatively new distribution of kidney damage. One was to define CKD solely by using the period of the hospitalization, i.e., without checking the history of CKD for the last 3 months. Thus, on the one hand, we did not miss any CKD patients, so all of them were included in the cohort, but on the other hand, patients without CKD might be included. Nevertheless, the high mortality of the CKD group was significantly higher than in the normal renal function group. 
Secondly, we used a classification of "AKI-RISE" and "AKI-DROP." We believed that these two groups must be separated because they might behave differently. For example, patients admitted to the hospital with a very high s-Cr level might have a better prognosis than those already treated in the hospital, and with the $\mathrm{s}-\mathrm{Cr}$ rise during hospitalization. Eventually, the difference in the mortality rate and different prognoses between the two groups was found. Moreover, we used a known RIFLE definition for AKI [8] - an elevation of s-Cr level $\geq 26.5(\mu \mathrm{mol} / \mathrm{L})$ indicates an AKI. However, we doubted some CKD patients, who had an extremely high s-Cr $(\geq 300[\mu \mathrm{mol} / \mathrm{L}])$ level, and whether this 26.5 rise would have been sufficient to reclassify them to the AKI group. Since this definition was used for all patients, we allocated the $8 \mathrm{CKD}$ patients with extremely high s-Cr $(300+)$ level into the AKI group. Nevertheless, we believe that this question should be faced and discussed in the future.

While performing the multivariate analysis on the comorbidities, it was rather surprising that the comorbidities were not significant for mortality risk. This finding is in controversy to other studies [12,15-17]. We assume that this finding is because we worked with a population of COVID-19 patients who came exclusively from the catchment area of our hospital, which is mainly inhabited by the senior population. It is supported by our results, too: the median age of all the patients in our study was 75 years. Therefore, we believe that due to the dominant elderly population and their multiple comorbidities, the influence of one single comorbidity on mortality was significantly reduced. This idea was supported by a recent article, which proved that the association between hypertension, diabetes, CVD, and COVID-19 indeed declined by an increase of age [18]. An additional explanation is that renal damage can serve as a marker (end-organ involvement) of other diseases' severity (diabetes, hypertension, or other comorbidities). It may explain why renal damage may be a better prognostic marker than the diagnosis of either of these comorbidities per se, which includes patients with mild condition.

On the contrary, we found gender, age, and renal damage as significant parameters for mortality of COVID-19 disease. This finding brought us to test the hypothesis of whether the eGFR value could be used to predict mortality from COVID-19 independently of the type of current renal damage and any other clinical parameter. The rationale behind it is that the eGFR formula is composed of s-Cr, age, gender, and ethnicity. Using eGFR values at admission, we classified the patients into 4 groups for mortality risk. The increased risk of mortality was in ac-

Mortality of Hospitalized COVID-19

Patients and Renal Parameters cordance with decreased eGFR in the groups. Also, contrary to the formation of 2 groups of mortality prediction used by other authors [19], the eGFR values distributed into 4 groups may predict the patients' prognosis more precisely on the first day of admission. However, we wanted to ensure that eGFR is the best predictive tool compared to other factors such as inflammatory markers. For this purpose, we used CRP and compared it to eGFR. Even though there are multiple other inflammatory markers, we decided to use CRP in particular because it was routinely available (no missing data were present), and it was measured mostly on the same day as the eGFR. Additionally, even though COVID is a viral infection, CRP was still proven as an inflammatory marker in patients suffering from COVID-19 [12]. Our findings helped us understand that eGFR is a better predictor than an inflammatory marker such as CRP.

Based on our results, we believe that eGFR on admission can indicate the survival prognosis, and it can be used as a simple and easily accessible parameter. It is particularly important when poor knowledge about patients' history and comorbidities is frequently a matter of truth at hospital admission.

The issue of COVID-19 prognostic scoring was already evaluated in several studies. Most of the authors analyzed different pneumonia severity markers [20-22], different laboratory markers, including inflammation ones [23-25], some renal parameters such as s-Cr levels development, albuminuria, or AKI course [24, 26], and some clinical parameters [21]. However, the results are somewhat difficult to summarize for an easy recommendation. Furthermore, no attention in a single study was given to how to evaluate the mortality risk at the time of hospital admission. To have such a marker available certainly can modify the treatment effort in these patients. Thus, we believe that our results concerning the eGFR mentioned above bring a real novelty in this matter.

Moreover, s-Cr level fluctuation during hospitalization may help to further reclassify patients according to their mortality risk. If a patient has an initial moderate and severe risk for mortality (grades 3b, 4, and 5), a drop in s-Cr level during hospitalization is associated with improved prognosis. However, for a patient at a mild risk group (grades 2 and $3 \mathrm{a}$ ) and s-Cr level rises, the patient's chance for mortality doubles. If the s-Cr level in the same group drops during the hospitalization or stays stable, there is no significant change in the mortality. The importance of s-Cr monitoring, particularly in the presence of renal damage, was already stressed by others $[21,27$, 28]. 
We also evaluated the relation between proteinuria and/or hematuria and mortality. These results are of interest since we did not find that these items were studied extensively in the literature. Nevertheless, the relation between COVID-19 and proteinuria has already been discussed, which may be caused by disruptions of glomerular capillaries and by tubular alterations [29]. Some studies used a strip test to show an association between proteinuria and mortality [30], but they did not distinguish it according to groups. Our study used a more extensive group, confirmed this correlation, and showed that it was independent of renal function. For receiving those results, we had to use the semiquantitative method (strip test) on admission and not a quantitative one (g/24 h), due to several reasons. Firstly, the strip test was routinely available and could be easily performed. Secondly, it would be challenging to collect urine for $24 \mathrm{~h}$ due to some patients' poor health conditions. The only reasonable option to do it was by placing a urinary catheter, which, in our study, was inserted only into patients directly admitted to the ICU. Therefore, studies used urinary protein-creatinine ratio and other semiquantitative analyses as the only appropriate option for hematuria and proteinuria analysis $[22,31,32]$. In contrast to our proteinuria results, only the CKD group displayed a correlation with the mortality for hematuria. Our findings intensify the understanding that COVID-19 can damage the kidneys, form proteinuria, and increase mortality risk.

Although we are aware of some limitations of our study, we believe that the core results would not be changed. First, we were not able to include ethnicity as an additional factor in the eGFR calculator. All the patients admitted to the hospital during the COVID-19 were Caucasian but not African American or Asian origin since their percentile in the population in Prague is very low. Thus, our result should be limited only to the Caucasian population. Second, we did not enter laboratory parameters, such as white blood cell count and serum albumin levels. The main reason for that, as explained above, was our will to concentrate on simple and readily available lab testing. Third, the strip data on proteinuria/hematuria were available only for two-thirds of enrolled patients. Furthermore, we could not correlate strip values with quantitative values (e.g., urinalysis or urinary albumin/ creatinine ratio).

In conclusion, the most important novelty, useful for common practice, is that we proved that calculating the eGFR on admission can be an excellent tool for predicting mortality, independently of any present renal damage. Besides that, the change in s-Cr levels during hospitaliza- tion reflects and further enhances the mortality prognosis. Additionally, as has already been discussed in the past, we confirmed that any kind of renal damage (AKI or CKD) significantly increases mortality risk in patients requiring hospitalization due to COVID-19. However, we proved it on a much more detailed scale and reflected "real-life conditions" in an emergency setting.

\section{Acknowledgment}

The authors would like to thank Dr. Marian Rybáŕ, MHA, for his statistical advice.

\section{Statement of Ethics}

Prior to starting the study's design, methodology, data collection, and data reporting, the full study protocol was approved by the FNKV Ethics Committee named "Eticke Komise Fakultni Nemocnice Karlovske Vinohrady." The decision reference number is EK-VP/70/0/2020. The study design was retrospective, so we used anonymized extracted data. Therefore, written informed consent was not required and waived by the "Eticke Komise Fakultni Nemocnice Karlovske Vinohrady" (EK-VP/70/0/2020).

\section{Conflict of Interest Statement}

The authors have no conflicts of interest to declare.

\section{Funding Sources}

There are no funders to report for this submission.

\section{Author Contributions}

E.Z. and A.S. contributed equally; E.Z., A.S., M.H., and I.R. substantially contributed to the conception and design of the study, performed the analysis and interpretation of data, and drafted and revised the work; K.J., K.K., and N.U. contributed to the conception of the study, collected data, and revised the work critically.

\section{Data Availability Statement}

The data that support the findings of this study are not publicly available due to information that could compromise the privacy of research participants, but are available from Prof. Ivan Rychlík, MD, PhD (corresponding author). 


\section{References}

1 World Health organization. Pneumonia of unknown cause: China. Available from: https: //www.who.int/emergencies/disease-outbreak-news/item/2020-DON229 (accessed September 2, 2021).

2 World Health organization. WHO coronavirus (COVID-19) dashboard | WHO coronavirus (COVID-19) dashboard with vaccination data. Available from: https://covid19. who.int/ (accessed January 6, 2022).

3 ERA-EDTA Council; ERACODA Working Group. Chronic kidney disease is a key risk factor for severe COVID-19: a call to action by the ERA-edta. Nephrol Dial Transplant. 2021;36:87-94.

4 Portolés J, Marques M, López-Sánchez P, de Valdenebro M, Muñez E, Serrano ML, et al. Chronic kidney disease and acute kidney injury in the COVID-19 Spanish outbreak. Nephrol Dial Transplant. 2020;35:1353-61.

5 Gallo Marin B, Aghagoli G, Lavine K, Yang L, Siff EJ, Chiang SS, et al. Predictors of COVID-19 severity: a literature review. Reviews in Medical Virology. 2021;31:1-10.

6 Jiwa N, Mutneja R, Henry L, Fiscus G, Zu Wallack R. Development of a brief scoring system to predict any-cause mortality in patients hospitalized with COVID-19 infection. PLoS One. 2021;16(7):e0254580.

7 Levey AS, Stevens LA, Schmid CH, Zhang YL, Castro AF 3rd, Feldman HI, et al. A New equation to estimate glomerular filtration rate. Ann Int Med. 2009;150:604.

8 Srisawat N, Kellum JA. Acute kidney injury: definition, epidemiology, and outcome. Curr Opin Crit Care. 2011;17:548-55.

9 Levey AS, de Jong PE, Coresh J, El Nahas M, Astor BC, Matsushita K, et al. The definition, classification, and prognosis of chronic kidney disease: a KDIGO controversies conference report. Kidney Int. 2011;80:17-28.

$10 \mathrm{Xu} \mathrm{Y,} \mathrm{Chen} \mathrm{Y,} \mathrm{Tang} \mathrm{X.} \mathrm{Guidelines} \mathrm{for} \mathrm{the} \mathrm{di-}$ agnosis and treatment of coronavirus disease 2019 (COVID-19) in China. Global Health Med. 2020;2:66-72.

11 European Centre for Disease Prevention and Control. Treatment and pharmaceutical prophylaxis of COVID-19. Available from: https: //www.ecdc.europa.eu/en/covid-19/latestevidence/treatment (accessed September 2, 2021).
12 Li J, Huang DQ, Zou B, Yang H, Hui WZ, Rui F, et al. Epidemiology of COVID-19: a systematic review and meta-analysis of clinical characteristics, risk factors, and outcomes. J Med Virol. 2021;93:1449-58.

13 Fisher M, Neugarten J, Bellin E, Yunes M, Stahl L, Johns TS, et al. AKI in hospitalized patients with and without COVID-19: a Comparison Study. J Am Soc Nephrol. 2020; 31:2145-57.

14 Liu YF, Zhang Z, Pan XL, Xing GL, Zhang Y, Liu ZS, et al. The chronic kidney disease and acute kidney injury involvement in COVID-19 pandemic: a systematic review and meta-analysis. PLoS One;16:e0244779.

15 Hessami A, Shamshirian A, Heydari K, Pourali F, Alizadeh-Navaei R, Moosazadeh $\mathrm{M}$, et al. Cardiovascular diseases burden in COVID-19: systematic review and metaanalysis. Am J Emerg Med. 2021;46:382-91.

16 Aghili SMM, Ebrahimpur M, Arjmand B, Shadman Z, Pejman Sani M, Qorbani M, et al. Obesity in COVID-19 era, implications for mechanisms, comorbidities, and prognosis: a review and meta-analysis. Int J Obes. 2021;45: 998-1016.

17 Rabbani G, Shariful Islam SM, Rahman MA, Amin N, Marzan B, Robin RC, et al. Pre-existing COPD is associated with an increased risk of mortality and severity in COVID-19: a rapid systematic review and meta-analysis. Expert Rev Respir Med. 2021;15:705-16.

$18 \mathrm{Hu}$ J, Wang Y. The clinical characteristics and risk factors of severe COVID-19. Gerontology. 2021;67:255-66.

19 Zhang C, Qin L, Li K, Zhao Y, Xu B, Liang L, et al. A novel scoring system for prediction of disease severity in COVID-19. Front Cell Infect Microbiol. 2020;10:338.

20 de Almeida DC, Franco MDCP, Dos Santos DRP, Santos MC, Maltoni IS, Mascotte F, et al. Acute kidney injury: incidence, risk factors, and outcomes in severe COVID-19 patients. PLoS One. 2021;16(5):e0251048.

21 Maguire D, Woods M, Richards C, Dolan R, Veitch JW, Sim WMJ, et al. Prognostic factors in patients admitted to an urban teaching hospital with COVID-19 infection. J Transl Med. 2020;18(1):354.

22 Morell-Garcia D, Ramos-Chavarino D, Bauça JM, Argente Del Castillo P, Ballesteros-Vizoso MA, García de Guadiana-Romualdo L, et al. Urine biomarkers for the prediction of mortality in COVID-19 hospitalized patients. Sci Rep. 2020;11(1):11134.
23 Torres-Macho J, Ryan P, Valencia J, PérezButragueño M, Jiménez E, Fontán-Vela M, et al. The PANDEMYC score. An easily applicable and interpretable model for predicting mortality associated with COVID-19. J Clin Med. 2020;9:3066.

24 Bartoletti M, Giannella M, Scudeller L, Tedeschi S, Rinaldi M, Bussini L, et al. Development and validation of a prediction model for severe respiratory failure in hospitalized patients with SARS-CoV-2 infection: a Multicentre Cohort Study (PREDI-CO Study). Clin Microbiol Infect. 2020;26:1545-53.

25 Macias-Muñoz L, Wijngaard R, González-de la Presa B, Bedini JL, Morales-Ruiz M, Jiménez W. Value of clinical laboratory test for early prediction of mortality in patients with COVID-19: the BGM score. J Circ Biomark. 2021;10:1-8.

26 Zhang B, Liu Q, Zhang X, Liu S, Chen W, You $J$, et al. Clinical utility of a nomogram for predicting 30-days poor outcome in hospitalized patients with COVID-19: multicenter external validation and decision curve analysis. Front Med;7:590460.

27 Antunez Muiños PJ, López Otero D, AmatSantos IJ, López País J, Aparisi A, Cacho Antonio CE, et al. The COVID-19 lab score: an accurate dynamic tool to predict in-hospital outcomes in COVID-19 patients. Sci Rep. 2021;11(1):9361.

28 Chen B, Gu HQ, Liu Y, Zhang G, Yang H, Hu $\mathrm{H}$, et al. A model to predict the risk of mortality in severely ill COVID-19 patients. Comput Struct Biotechnol J. 2021;19:1694-700.

29 Mohamed MMB, Velez JCQ. Proteinuria in COVID-19. Clin Kidney J. 2021;14(Suppl 1): i40-7.

30 Huart J, Bouquegneau A, Lutteri L, Erpicum P, Grosch S, Résimont G, et al. Proteinuria in COVID-19: prevalence, characterization and prognostic role. J Nephrol. 2021;34:355-64.

31 Ouahmi H, Courjon J, Morand L, François J, Bruckert V, Lombardi R, et al. Proteinuria as a biomarker for COVID-19 severity. Front Physiol. 2021;12:611772.

32 Jafari-Oori M, Fiorentino M, Castellano G, Ebadi A, Rahimi-Bashar F, Guest PC, et al. Acute kidney injury and covid-19: a scoping review and meta-analysis. Adv Exp Med Biol. 2021;1321:309-24.
Mortality of Hospitalized COVID-19

Patients and Renal Parameters
Kidney Blood Press Res 2022;47:309-319 DOI: $10.1159 / 000522100$ 